TITLE:

\title{
Parameter optimization of tetrahedral tuned mass damper for three-directional seismic response reduction
}

\section{$\operatorname{AUTHOR}(S)$ :}

Ohsaki, Makoto; Tsuda, Seita; Hasegawa, Toma

\section{CITATION:}

Ohsaki, Makoto ...[et al]. Parameter optimization of tetrahedral tuned mass damper for three-directional seismic response reduction. Engineering Structures 2016, 126: 667-674

\section{ISSUE DATE:}

2016-11-01

URL:

http://hdl.handle.net/2433/229536

\section{RIGHT:}

(C)2016. This manuscript version is made available under the CC-BY-NC-ND 4.0 license

http://creativecommons.org/licenses/by-nc-nd/4.0/; The full-text file will be made open to the public on 1 November 2018 in accordance with publisher's 'Terms and Conditions for Self-Archiving'.; この論文は出版社版でありません。引 用の際には出版社版をご確認ご利用ください。;This is not the published version. Please cite only the published version. 


\title{
Parameter Optimization of Tetrahedral Tuned Mass Damper for Three-directional Seismic Response Reduction
}

\author{
Makoto Ohsaki ${ }^{1 *}$, Seita Tsuda ${ }^{2}$, Toma Hasegawa ${ }^{3}$ \\ ${ }^{1}$ Department of Architecture and Architectural Engineering, Graduate School of \\ Engineering, Kyoto University, Japan,ohsaki@archi.kyoto-u.ac.jp \\ ${ }^{2}$ Department of Design and Technology, Okayama Prefectural University, Japan \\ ${ }^{3}$ Department of Architecture, Graduate School of Engineering, Hiroshima University, \\ Japan
}

\begin{abstract}
An optimization approach is presented for design of a tetrahedral tuned mass damper called TD-TMD for three-directional seismic response reduction of structures. The mass damper consists of a viscous damper and a mass connected by springs and a rigid bar. By utilizing flexibility of the springs, movement of the mass in three-directions and elongation of the viscous damper are amplified, and vibration energy of the structure is effectively dissipated by the viscous damper. The objective function of the parameter optimization problem is the mean norm of the response displacements of the structure. The bounds of parameters are determined by solving an auxiliary nonlinear programming problem to maximize the minimum deformation of the damper against unit static loads in various directions. Approximate optimal solutions are found using a heuristic approach called simulated annealing combined with pure random search that generates efficient intial solutions. The TD-TMD is attached to a simple three-degree-of-freedom structure, and the seismic responses are compared with those with conventional single-directional tuned mass dampers.
\end{abstract}

Keywords: Tuned mass damper, Parameter optimization, Seismic response, Multicomponent motion.

\section{INTRODUCTION}

Among various devices for passive control of vibration, tuned mass damper (TMD) is effectively used for reduction of vibration due to seismic and/or wind excitations. However, a conventional TMD can reduce the responses in a single direction dominated 
by a single mode of vibration. Therefore, several TMDs are needed for reduction of multidirectional and multi-modal vibrations [1,2]. Viet and Nghi [3] proposed a two-mode control device utilizing geometrically nonlinear effect of a spring and a pendulum. Ueng et al. [4] proposed a design method of multiple TMDs for buildings under torsional vibration. Yoshinaka and Kawaguchi [5] investigated performances of multiple TMDs for vibration control of long span structures. The authors presented a mass damper that can reduce two-directional vibration of an arch using single mass and a viscous damper [6,7]. Almazán et al. [8] proposed a bi-directional TMD supporting a mass by vertical cables and bars.

Design of TMDs is strongly related to optimization, because the parameters of TMDs should be appropriately tuned so that the vibration of the mass is amplified by the vibration of the structure. De Angelis et al. [9] optimized the parameters of a large-massratio TMD that is connected to the structure using a high-damping rubber bearing. Lavan and Daniel [10] optimized the locations and parameters of multiple TMDs for a building frame with irregular plan. Lin et al. [11] assigned an upper bound for the stroke for tuning the parameters. Garrid et al. [12] used a computational approach to optimize the TMD with rotational degree of freedom. Heuristic approaches such as harmony search [13] and particle swarm optimization [14] are also used for optimizing TMDs.

In this paper, a mass damper called three-directional TMD (TD-TMD) is proposed for reduction of three-directional vibration of a structure subjected to multi-component ground motions. The mass damper has a tetrahedral shape consisting of a viscous damper and a mass connected by springs and a rigid bar. By utilizing the flexibility of springs, the movement of the mass in three-directions and the elongation of viscous damper are amplified, and the vibration energy of the structure is effectively dissipated by the viscous damper. The variables consisting of stiffnesses of the springs, damping coefficient of the viscous damper, and locations of the nodes are discretized into integer values, and their optimal values are found using a global pure random search (PRS) $[15,16]$ combined with a heuristic method of local search called simulated annealing (SA) [17,18]. Effectiveness of optimizing TD-TMD is demonstrated by comparing the response reduction properties of a simple three-degree-of-freedom (3DOF) structure with those of the conventional TMDs with the same total mass.

\section{Description of TMD models and seismic motions}

\subsection{TD-TMD model}

Figure 1 illustrates the proposed TD-TMD, which can reduce the three-directional vibration using a single set of mass and viscous damper. The TD-TMD has a tetrahedral 
shape, and a mass $M$ is located at node $\mathrm{D}$, which is connected to nodes $\mathrm{A}$ and $\mathrm{C}$ by the springs with the extensional stiffness $K_{2}$, and $K_{3}$, respectively. Node A is attached to the structure, and node $\mathrm{C}$ has the same three-directional displacements as node $\mathrm{A}$. Node $\mathrm{B}$ has the same $X$ - and $Y$-directional displacements as node $\mathrm{A}$, and is free in $Z$-direction so that the viscous damper with the damping ratio $C_{1}$ can deform in accordance with vibration of the mass at node $\mathrm{D}$. Furthermore, nodes $\mathrm{B}$ and $\mathrm{D}$ are connected by a rigid bar.

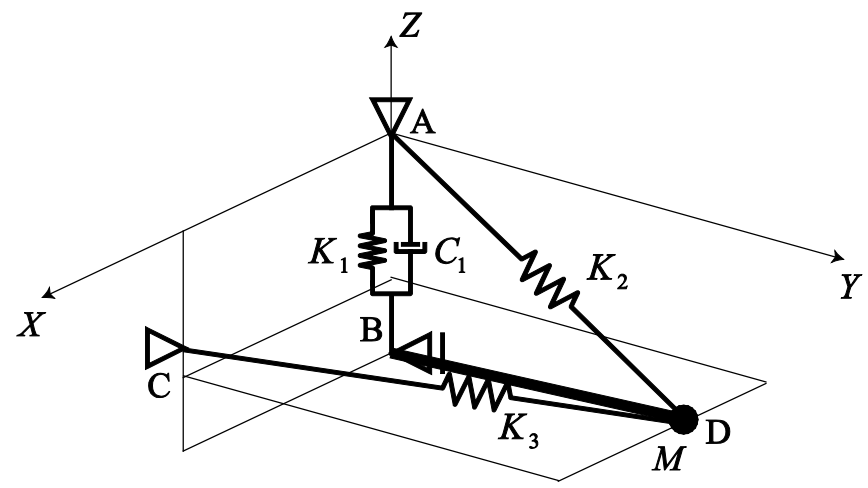

Figure 1: Components of TD-TMD; a mass at node D, vertical viscous damper between nodes $\mathrm{A}$ and $\mathrm{B}$, three springs, and a rigid bar.

Springs with small extensional stiffness $K_{1}$ is added between nodes A and B, and a small mass is attached at node B to stabilize the device. We assume that the tetrahedral device is contained in a box so that it can be attached to the structure easily. The threedirectional vibration of node $\mathrm{D}$ leads to deformation of the viscous damper between nodes $\mathrm{A}$ and $\mathrm{B}$, which dissipates seismic energy. By tuning the parameters of the TD-TMD, three-directional vibration of the structure can be reduced by a pair of mass and viscous damper.

\subsection{SD-TMD model}

We compare the vibration reduction property of the TD-TMD with that of the singledirectional TMD (SD-TMD), which consists of a mass connected by a spring and a viscous damper. Three SD-TMDs called dampers 1, 2, and 3 are installed, as shown in Fig. 2, for vibration reduction in $X$-, $Y$-, and $Z$-directions, respectively. Node $\mathrm{A}$ is to be attached at the structure. The mass, extensional stiffness of spring, and damping 
coefficient of damper $i(=1,2,3)$ are denoted by $m_{i}, k_{i}$, and $c_{i}$, respectively. When comparing the performances of TD-TMD and SD-TMDs, the total value of $m_{1}, m_{2}$, and $m_{3}$ is the same as the mass of TD-TMD; i.e., $m_{1}+m_{2}+m_{3}=M$.

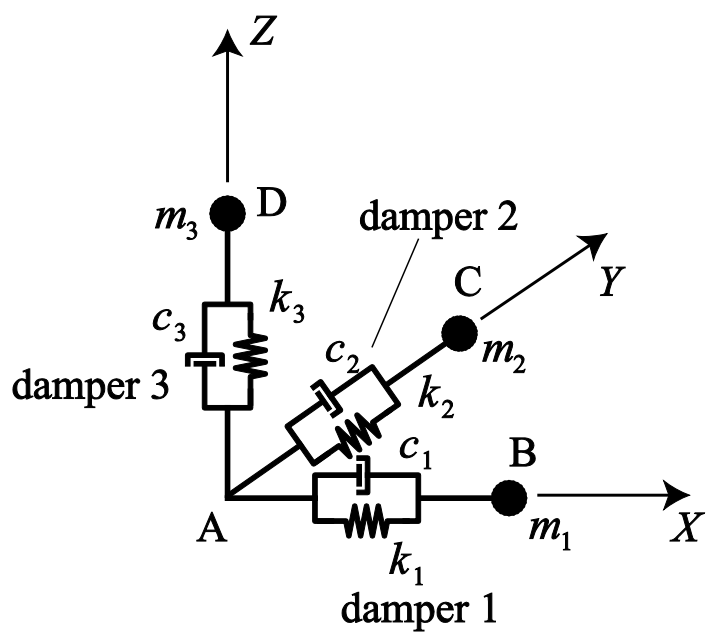

Figure 2: A set of three SD-TMDs for vibration control in three-directions.

The parameters of dampers 1, 2, and 3 are adjusted to reduce the responses in each direction. Let $M_{i}$ denote the equivalent mass of the dominant mode $\boldsymbol{\Phi}_{i}$ of a structure, for which the vibration is to be reduced by damper $i$ in $X-, Y$-, or $Z$-direction. The mass ratio is given as $\mu_{i}=m_{i} / M_{i} \quad(i=1,2,3)$. The frequency ratios $\gamma_{i}$ and damping factors $\xi_{i} \quad(i=1,2,3)$ of dampers 1,2 , and 3 are determined using the following optimal values of SD-TMD against various dynamic loading conditions [19]:

- Case 1: Sinusoidal motion

$$
\gamma_{i}=\frac{1}{1+\mu_{i}}, \quad \xi_{i}=\sqrt{\frac{3 \mu_{i}}{8\left(1+\mu_{i}\right)}}
$$

- Case 2: Sinusoidal ground motion

$$
\gamma_{i}=\frac{1}{\sqrt{1+\mu_{i}}}, \quad \xi_{i}=\sqrt{\frac{3 \mu_{i}}{8\left(1+\mu_{i} / 2\right)}}
$$

- Case 3: Random ground motion 


$$
\gamma_{i}=\frac{\sqrt{1+\mu_{i} / 2}}{1+\mu_{i}}, \quad \xi_{i}=\frac{1}{2} \sqrt{\frac{\mu_{i}\left(1+3 \mu_{i} / 4\right)}{1+3 \mu_{i} / 2}}
$$

Let $\omega_{i}$ denote the natural circular frequency of mode $\boldsymbol{\Phi}_{i}$ of the structure. The optimal stiffness $\tilde{K}_{i}$ of the SD-TMD for controlling mode $\boldsymbol{\Phi}_{i}$ is computed from

$$
\tilde{K}_{i}=m_{i}\left(\gamma_{i} \omega_{i}\right)^{2}
$$

\subsection{Seismic motion}

Dynamic responses of the structure with TMDs are evaluated by time-history analysis using a software package called OpenSees Ver. 2.4 [20]. The design acceleration response spectrum is specified according to the Notification 1461 of Ministry of Land, Infrastructure, Transport, and Tourism (MLIT), Japan, corresponding to the performance level of operational limit for the "design based on calculation of response and limit state," which is similar to the capacity spectrum approach. The acceleration response spectrum $S_{\mathrm{A}}(T, h)$ is given with respect to the natural period $T$ and the damping factor $h$ as

$$
S_{\mathrm{A}}(T, h)=\frac{1.5}{1+10 h}\left\{\begin{array}{l}
0.96+9.0 T \text { for } T \leq 0.16 \\
2.4 \text { for } 0.16 \leq T \leq 0.864 \\
2.074 / T \text { for } 0.864 \leq T
\end{array}\right.
$$

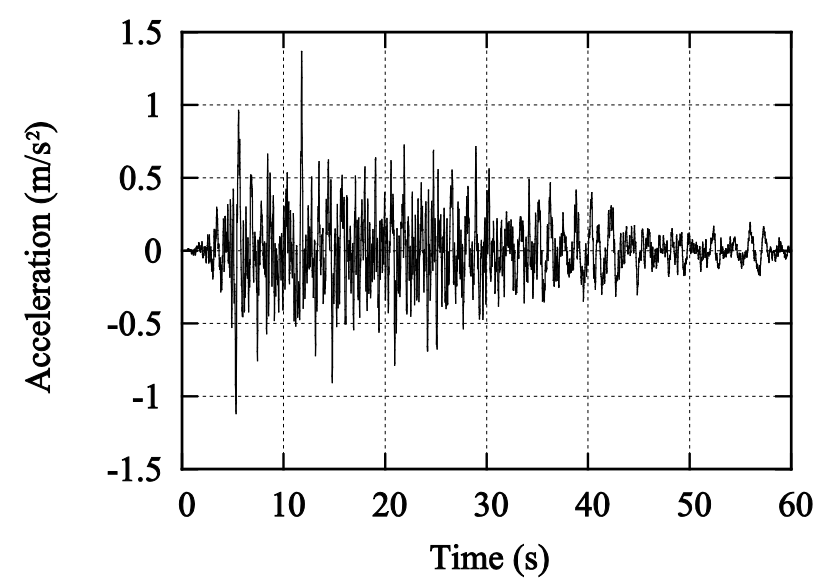

Figure 3: An example of ground motion. 
Five sets of ground motions are generated as combinations of ground motions compatible to the acceleration response sprctrum in Eq. (5). The ground motions in each direction are generated using a standard approach of superposition of sinusoidal waves with random phase [21,22]. The duration of ground motion is 60 seconds; however, only the response for the first 40 seconds is computed. The time step for integration is $0.01 \mathrm{~s}$.

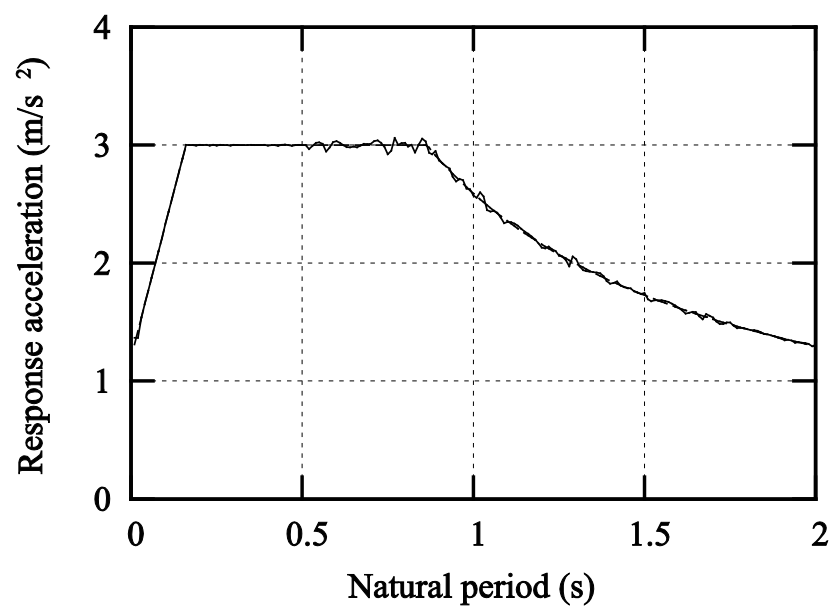

Figure 4: Acceleration response spectrum of ground motion in Fig. 3.

An example of time-history of acceleration and its acceleration response spectrum for $h=0.05$ are shown in Figs. 3 and 4, respectively. Different motions among the five are selected for $X$-, $Y$-, and $Z$-directions; therefore, the total number of sets is 60 . The acceleration in $Z$-direction is multiplied by 0.5 to incorporate practical situation.

\section{Structure models}

We consider a simple 3DOF structure as shown in Fig. 5 . The springs $K_{1}, K_{2}, K_{3}$, and the mass at node A represent the stiffness and mass $(1000 \mathrm{~kg})$ of the structure, which are specified so that the natural periods $T_{X}, T_{Y}$, and $T_{Z}$ in $X$-, $Y$-, and $Z$-directions, respectively, are $0.3,0.3$, and $0.45 \mathrm{~s}$. Rayleigh damping is used such that the damping factors are 0.02 for the $X$ - and $Z$-directional vibrations of the structure. 


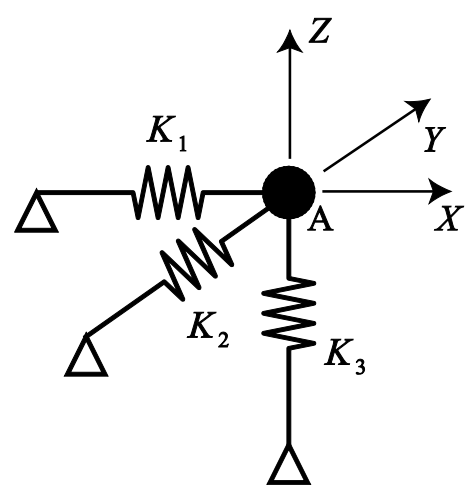

Figure 5: A simple 3DOF structure.

The mass of TD-TMD is $1 / 20$ and each mass of the SD-TMD is $1 / 60$ of the structural mass. The values of stiffness and damping ratio corresponding to Cases 1-3 (Eqs. (1) -(3)) of SD-TMD are listed in Table 1. Note that 'Opt.' in Table 1 is described in Sec. 4. The stiffness and damping ratio have the same values, respectively, for $X$ - and $Y$ directions, because the natural periods in $X$-and $Y$-directions of the structure are the same.

Table 1: Values of stiffness $(\mathrm{kN} / \mathrm{m})$ and damping ratio $(\mathrm{Ns} / \mathrm{m})$ of SD-TMDs.

\begin{tabular}{ccccc}
\hline & $k_{1}, k_{2}$ & $k_{3}$ & $c_{1}, c_{2}$ & $c_{3}$ \\
\hline Case 1 & 7.073 & 3.144 & 53.84 & 35.89 \\
\hline Case 2 & 7.132 & 3.270 & 44.24 & 29.49 \\
\hline Case 3 & 7.251 & 3.223 & 54.74 & 36.49 \\
\hline Opt. & 6.989 & 2.980 & 39.81 & 27.72 \\
\hline
\end{tabular}

\section{Optimization problems of TD-TMD}

\subsection{Formulation of objective function}

The parameters of TD-TMD in Fig. 1 are optimized for reducing the seismic responses of the structure. Let $X_{\mathrm{C}}, X_{\mathrm{D}}, Y_{\mathrm{D}}$, and $Z_{\mathrm{D}}$ denote the $X$-coordinates of nodes $\mathrm{C}, \mathrm{D}$, the $Y$ coordinate of node $\mathrm{D}$, and $Z$-coordinate of node $\mathrm{D}$, respectively. The difference between $Z$ coordinates of nodes $\mathrm{C}$ and $\mathrm{D}$, denoted by $Z_{\mathrm{CD}}=Z_{\mathrm{D}}-Z_{\mathrm{C}}$, is also considered as variable. In addition to these five geometrical parameters, the stiffnesses $K_{1}, K_{2}, K_{3}$ of the springs and the damping coefficient $C_{1}$ of the viscous damper are considered as variables; i.e., we 
have nine variables. The length of vertical damper is $1 \mathrm{~m}$, and the $X$-coordinate of node $\mathrm{C}$ is fixed at 0 . A small mass of $1 \mathrm{~kg}$ is placed at node $\mathrm{B}$ to stabilize the numerical analysis.

The five sets of seismic motions among 60 sets are used for optimization. Small deformation is assumed in the process of optimization. Let $x_{i}, y_{i}$, and $z_{i}$ denote the $X$-, $Y$-, and $Z$-directional displacements at node $\mathrm{A}$ of the structure at the $i$ th step of analysis. The total number of analysis steps is denoted by $N$. The mean values $D_{X}, D_{Y}, D_{Z}$, and $D_{X Y Z}$ of squares of nodal displacements are defined by

$$
\begin{array}{ll}
D_{X}=\frac{1}{N} \sum_{i=1}^{N}\left(x_{i}\right)^{2}, & D_{Y}=\frac{1}{N} \sum_{i=1}^{N}\left(y_{i}\right)^{2}, \\
D_{Z}=\frac{1}{N} \sum_{i=1}^{N}\left(z_{i}\right)^{2}, & D_{X Y Z}=D_{X}+D_{Y}+D_{Z}
\end{array}
$$

The reduction ratios $R_{X}, R_{Y}, R_{Z}$, and $R_{X Y Z}$ are defined, respectively, as the ratios of $D_{X}, D_{Y}, D_{Z}$, and $D_{X Y Z}$ of the structure with TD-TMD to the values without TMD. Let $\mathbf{d}, \mathbf{d}^{\mathrm{L}}$, and $\mathbf{d}^{\mathrm{U}} \in \mathbb{R}^{9}$ denote the vector consisting of nine variables and their lower and upper bounds, respectively. The optimization problem is defined as follows for minimizing the mean value $R_{X Y Z}^{\text {mean }}(\mathbf{d})$ of $R_{X Y Z}(\mathbf{d})$ among the five sets of motions:

$$
\begin{array}{ll}
\text { Minimize } & R_{X Y Z}^{\text {mean }}(\mathbf{d}) \\
\text { subject to } & \mathbf{d}^{\mathrm{L}} \leq \mathbf{d} \leq \mathbf{d}^{\mathrm{U}}
\end{array}
$$

\subsection{Determination of ranges of variables of TD-TMD solving static optimization problem}

The bounds of variables except the damping coefficient of TD-TMD are determined by solving an auxiliary optimization problem based on static properties. Let $U_{1}, U_{2}$, and $U_{3}$ denote the displacements in $X$-, $Y$-, and $Z$-directions against the load $P$ in $X$-, $Y$-, and Z-directions, respectively, at node D of a TD-TMD. A vector consisting of eight 
variables $K_{1}, K_{2}, K_{3}, X_{\mathrm{C}}, X_{\mathrm{D}}, Y_{\mathrm{D}}, Z_{\mathrm{D}}$, and $Z_{\mathrm{CD}}$ is denoted by $\mathbf{d}_{\mathrm{S}} \in \mathbb{R}^{8}$. The static stiffness $K_{i}^{\mathrm{S}}\left(\mathbf{d}_{\mathrm{S}}\right)$ of the TD-TMD is evaluated as

$$
K_{i}^{\mathrm{S}}\left(\mathbf{d}_{\mathrm{S}}\right)=\frac{P}{U_{i}\left(\mathbf{d}_{\mathrm{S}}\right)}, \quad(i=1,2,3)
$$

The eight variables of TD-TMD are determined to satisfy the constraint $K_{i}^{\mathrm{S}}\left(\mathbf{d}_{\mathrm{S}}\right)=\tilde{K}_{i}$ $(i=1,2,3)$, where $\tilde{K}_{i}$ is computed from Eq. (4) using the frequency ratios $\gamma_{i}$ of Case 3.

Furthermore, the viscous damper of TD-TMD should have enough deformation for motion in any direction of the mass at node $\mathrm{D}$. Therefore, we maximize the minimum absolute value $e^{\min }\left(\mathbf{d}_{\mathrm{S}}\right)$ of the extensions of the damper against unit static loads at node $\mathrm{D}$ in various directions. The auxiliary static optimization problem is formulated as

$$
\begin{array}{ll}
\text { Maximize } & e^{\min }\left(\mathbf{d}_{\mathrm{S}}\right) \\
\text { subject to } & K_{i}^{\mathrm{S}}\left(\mathbf{d}_{\mathrm{S}}\right)=\tilde{K}_{i}, \quad(i=1,2,3) \\
& \mathbf{d}_{\mathrm{S}}^{\mathrm{L}} \leq \mathbf{d}_{\mathrm{S}} \leq \mathbf{d}_{\mathrm{S}}^{\mathrm{U}}
\end{array}
$$

where $\mathbf{d}_{\mathrm{S}}^{\mathrm{L}}$ and $\mathbf{d}_{\mathrm{S}}^{\mathrm{U}}$ are the lower and upper bounds for $\mathbf{d}_{\mathrm{S}}$.

\section{Optimization results}

\subsection{Result of auxiliary static optimization of TD-TMD}

Problem (9) is solved to determine the bounds of variables for the dynamic optimization problem (7). The load of magnitude $P=10.0(\mathrm{kN})$ is applied at node D of TD-TMD in thirteen different directions as shown in Table 2. The optimization library SNOPT Ver. 7 [23] is used for solving the auxiliary optimization problem.

Based on the preliminary investigation, the lower and upper bounds of the variables are given as listed in the first and second rows of Table 3. The rigid bar is modeled as a stiff spring with the extensional stiffness $100 \mathrm{kN} / \mathrm{m}$. Local optimal solutions are found from ten different initial solutions, which are generated randomly. The best solution that has the maximum objective function value among the ten local optimal solutions is listed in the last row of Table 3 . The absolute value of damper extension, which is the objective 
function of Problem (9), of the optimal solution in Table 3 is listed in the last column of Table 2 for each load case of the optimal solutions.

Table 2: Directions of thirteen loads and corresponding absolute value of damper extensions (objective function values) of optimal solution.

\begin{tabular}{ccc}
\hline Load No. & Load direction & $\begin{array}{c}\text { Absolute value of damper } \\
\text { extension }(\mathrm{m})\end{array}$ \\
\hline 1 & $(1,0,0)$ & 1.3285 \\
2 & $(0,1,0)$ & 2.5070 \\
3 & $(0,0,1)$ & 1.9177 \\
4 & $(\sqrt{2}, \sqrt{2}, 0)$ & 2.7121 \\
5 & $(\sqrt{2},-\sqrt{2}, 0)$ & 0.8333 \\
6 & $(\sqrt{2}, 0, \sqrt{2})$ & 2.2954 \\
7 & $(\sqrt{2}, 0,-\sqrt{2})$ & 0.4167 \\
8 & $(0, \sqrt{2}, \sqrt{2})$ & 3.1287 \\
9 & $(0, \sqrt{2},-\sqrt{2})$ & 0.4167 \\
10 & $(\sqrt{3}, \sqrt{3}, \sqrt{3})$ & 3.3216 \\
11 & $(-\sqrt{3}, \sqrt{3}, \sqrt{3})$ & 1.7876 \\
12 & $(\sqrt{3},-\sqrt{3}, \sqrt{3})$ & 0.4268 \\
13 & $(\sqrt{3}, \sqrt{3},-\sqrt{3})$ & 1.1072 \\
\hline
\end{tabular}

Table 3: Bounds of variables and optimal solution of auxiliary static optimization problem (9).

\begin{tabular}{ccccc}
\hline & $\begin{array}{c}K_{1} \\
(\mathrm{kN} / \mathrm{m})\end{array}$ & $\begin{array}{c}K_{2} \\
(\mathrm{kN} / \mathrm{m})\end{array}$ & $\begin{array}{c}K_{2} \\
(\mathrm{kN} / \mathrm{m})\end{array}$ & $X_{\mathrm{C}}(\mathrm{m})$ \\
\hline Lower bound & 0.5 & 1.0 & 1.0 & 0.5 \\
$\begin{array}{c}\text { Upper bound } \\
\text { Optimal solution }\end{array}$ & 0.52145 & 100.0 & 100.0 & 1.5 \\
& $X_{\mathrm{D}}(\mathrm{m})$ & $Y_{\mathrm{D}}(\mathrm{m})$ & $Z_{\mathrm{D}}(\mathrm{m})$ & $Z_{\mathrm{CD}}(\mathrm{m})$ \\
\hline & 0.3 & 0.2 & -0.7 & -0.2 \\
& 0.7 & 1.0 & -0.5 & 0.2 \\
& 0.3000 & 0.5295 & -0.7000 & 0.2000 \\
\hline
\end{tabular}


Table 4: Bounds of variables and optimal solution of problem (7).

\begin{tabular}{cccccc}
\hline & $\begin{array}{c}K_{1} \\
(\mathrm{kN} / \mathrm{m})\end{array}$ & $\begin{array}{c}K_{2} \\
(\mathrm{kN} / \mathrm{m})\end{array}$ & $\begin{array}{c}K_{3} \\
(\mathrm{kN} / \mathrm{m})\end{array}$ & $X_{\mathrm{C}}(\mathrm{m})$ & $X_{\mathrm{D}}(\mathrm{m})$ \\
\hline Lower bound & 0.5 & 5.0 & 5.0 & 0.5 & 0.3 \\
Upper bound & 5.0 & 50.0 & 50.0 & 1.5 & 0.7 \\
Optimal solution & 1.625 & 17.150 & 19.400 & 1.25 & 0.66 \\
\hline & $Y_{\mathrm{D}}(\mathrm{m})$ & $Z_{\mathrm{D}}(\mathrm{m})$ & $Z_{\mathrm{CD}}(\mathrm{m})$ & $C_{1}$ & \\
& & & & $(\mathrm{Ns} / \mathrm{m})$ & \\
\hline & 0.2 & -0.7 & -0.2 & 50.0 & \\
& 1.0 & -0.5 & 0.2 & 500.0 & \\
& 0.728 & -0.583 & -0.060 & 115.25 & \\
\hline
\end{tabular}

\subsection{Optimization result of TD-TMD for seismic response reduction}

From the result of auxiliary static optimization problem, the lower and upper bounds of the variables except $C_{1}$ for problem (7) are assigned as listed in Table 4. Since the viscous damper of the TD-TMD is not connected directly between the mass and structure, the movement of the mass does not directly lead to the extension of the viscous damper. Furthermore, the TD-TMD has only one viscous damper, and the mass of TD-TMD is three times as large as that of an SD-TMD. Therefore, a larger damping ratio is needed for TD-TMD than SD-TMD, and the bounds of $C_{1}$ are determined, as shown in Table 4, from the results of preliminary seismic response analysis.

We solve problem (7) using heuristic approaches of combinatorial problem. Therefore, the nine variables $d_{1}, \ldots, d_{9}$ are discretized into 21 equally spaced values $d_{i}^{r}$ between the lower bound $d_{i}^{\mathrm{L}}$ and upper bound $d_{i}^{\mathrm{U}}$ as

$$
d_{i}^{r}=d_{i}^{\mathrm{L}}+\frac{r-1}{20}\left(d_{i}^{\mathrm{U}}-d_{i}^{\mathrm{L}}\right), \quad(r=1, \ldots, 21)
$$


Since the problem considered here is highly nonlinear, 5000 sets of variables are randomly generated to carry out PRS $[15,16]$. The ten best solutions obtained by PRS are listed in Table 5, where 'Solution' corresponds to the integer value of $r$ in Eq. (10). Note that the objective function to be minimized is $R_{X Y Z}$; however, $R_{X}, R_{Y}$, and $R_{Z}$ are also listed to check the properties in each direction. As seen from Table 5, the responses in all three directions are successfully reduced by optimizing the TD-TMD.

Table 5: Ten best solutions obtained by PRS.

\begin{tabular}{clcccc}
\hline Order & Solution & $R_{X}$ & $R_{Y}$ & $R_{Z}$ & $R_{X Y Z}$ \\
\hline 1 & $7,7,7,17,21,14,12,7,3$ & 0.5551 & 0.5036 & 0.3245 & 0.4380 \\
2 & $2,8,9,16,18,19,21,10,17$ & 0.4626 & 0.5903 & 0.3538 & 0.4489 \\
3 & $20,5,11,17,2,14,17,13,6$ & 0.4647 & 0.6820 & 0.3391 & 0.4693 \\
4 & $12,5,7,21,13,10,13,13,9$ & 0.5282 & 0.4755 & 0.4331 & 0.4696 \\
5 & $1,7,9,17,11,17,15,10,14$ & 0.4685 & 0.6106 & 0.3927 & 0.4716 \\
6 & $19,7,9,16,18,19,4,21,19$ & 0.5280 & 0.6283 & 0.3367 & 0.4721 \\
7 & $2,6,7,10,16,10,18,10,12$ & 0.5323 & 0.4458 & 0.4684 & 0.4750 \\
8 & $8,6,9,14,3,16,16,16,21$ & 0.4473 & 0.5554 & 0.4618 & 0.4780 \\
9 & $21,5,10,9,8,13,5,17,19$ & 0.4289 & 0.5960 & 0.4465 & 0.4783 \\
10 & $1,7,10,16,18,18,19,9,5$ & 0.4008 & 0.7162 & 0.3850 & 0.4795 \\
\hline
\end{tabular}

Optimal solutions are also found for other sets of natural periods $T_{X}, T_{Y}$, and $T_{Z}$ in $X$-, $Y$-, and $Z$-directions of the structure. The reduction ratios for three cases obtained by PRS are listed in Table 6 . Note that the same bounds listed in Table 4 are used without solving the auxiliary static optimization problem. Therefore, a better solution than those in Table 6 may exist for each case. It is seen from Table 6 that the response in Z-direction is not reduced, if $T_{Z}$ has an intermediate value between $T_{X}$ and $T_{Y}$. This is because the magnitude of input motion in $Z$-direction is half of those in $X$ - and $Y$-directions; accordingly, only the responses in $X$ - and $Y$-directions are reduced to have smaller norm of response displacement.

Table 6: Solutions obtained by PRS for various natural periods.

\begin{tabular}{lllllll}
\hline$T_{X}$ & $T_{Y}$ & $T_{Z}$ & $R_{X}$ & $R_{Y}$ & $R_{Z}$ & $R_{X Y Z}$ \\
\hline
\end{tabular}




\begin{tabular}{lllllll}
\hline 0.35 & 0.45 & 0.40 & 0.4709 & 0.3723 & 1.3880 & 0.4786 \\
0.30 & 0.40 & 0.50 & 0.4571 & 0.6333 & 0.4534 & 0.5370 \\
0.35 & 0.30 & 0.40 & 0.5882 & 0.4919 & 0.5139 & 0.5302 \\
\hline
\end{tabular}

The ten solutions for $T_{X}=0.3, T_{Y}=0.3, T_{Z}=0.45(\mathrm{~s})$ in Table 5 are further improved using SA [17,18], which is a statistical extension of local search. Nine neighborhood solutions are generated randomly at each step of SA, and the best solution among them are selected as the candidate for the seed solution at the next step. The initial temperature parameter is defined such that the probability of acceptance of the solution is 0.5 when the increase of the objective function is 0.1 at the initial step. The temperature parameter is multiplied by 0.95 at each step. The total number of steps is 100 .

The initial solutions for SA are the ten best solutions in Table 5 obtained by PRS. The range of each variable is reduced to $1 / 10$ of the value in Table 4 for PRS with the nominal value equal to the optimal solution of PRS. Note that the lower or upper bound for SA is equal to the optimal value of PRS, if it is equal to lower or upper bound, respectively, for PRS in Table 4. The ten best solutions improved by SA are listed in Table 7. Note that 'Solution' corresponds to the integer value for the reduced range of variables. The values of variables of the best solution in Table 6 are listed in the last row of Table 4 .

Table 7: Ten improved solutions by SA.

\begin{tabular}{clcccc}
\hline Order & \multicolumn{1}{c}{ Solution } & $R_{X}$ & $R_{Y}$ & $R_{Z}$ & $R_{X Y Z}$ \\
\hline 1 & $1,5,15,1,1,13,18,21,20$ & 0.4913 & 0.4657 & 0.3253 & 0.4096 \\
2 & $20,3,2,21,7,1,21,21,1$ & 0.4917 & 0.5164 & 0.3438 & 0.4322 \\
3 & $1,19,1,13,21,21,1,21,21$ & 0.4056 & 0.6628 & 0.3136 & 0.4364 \\
4 & $10,13,7,3,20,21,13,1,1$ & 0.4911 & 0.4587 & 0.3359 & 0.4134 \\
5 & $21,4,1,10,21,11,21,21,1$ & 0.4772 & 0.5072 & 0.3520 & 0.4285 \\
6 & $1,8,16,1,1,1,21,21,1$ & 0.4856 & 0.6186 & 0.3343 & 0.4559 \\
7 & $21,5,16,20,21,21,5,21,1$ & 0.5122 & 0.4571 & 0.3832 & 0.4376 \\
8 & $21,4,4,21,21,1,10,21,1$ & 0.4671 & 0.4872 & 0.4083 & 0.4428 \\
9 & $5,9,1,21,21,4,21,21,1$ & 0.4520 & 0.5165 & 0.4030 & 0.4446 \\
10 & $21,12,1,21,13,8,1,2,21$ & 0.4162 & 0.6165 & 0.3459 & 0.4397 \\
\hline
\end{tabular}

Optimal solutions are also found for SD-TMD by discretizing each variable into 21 values. The lower and upper bounds of $k_{i} \quad(i=1,2,3)$ are $90 \%$ and $110 \%$ of the value 
of Case 2 in Table 1, while those for $c_{i}$ are $80 \%$ and $100 \%$ of the value of Case 2 in Table 1. Since the response in each direction is independent of those in other directions, and the optimal parameter values in $X$ - and $Y$-directions have the same values, the two sets $\left(k_{1}, c_{1}\right)$ and $\left(k_{3}, c_{3}\right)$ are optimized individually by enumeration of $21 \times 21=441$ possible pairs of values. The optimal values obtained by enumeration are listed in the last row of Table 1, which shows that the stiffness and damping coefficient are smaller than the values of all Cases $1-3$.

Table 8: Response reduction ratios for 60 sets of motions.

\begin{tabular}{|c|c|c|c|c|c|}
\hline & & Mean & Maximum & Minimum & Standard deviation \\
\hline \multirow{4}{*}{ TD-TMD } & $R_{X}$ & 0.5017 & 0.9601 & 0.3022 & 0.15223 \\
\hline & $R_{Y}$ & 0.5473 & 0.9028 & 0.2990 & 0.14874 \\
\hline & $R_{Z}$ & 0.3277 & 0.4268 & 0.2534 & 0.04285 \\
\hline & $R_{X Y Z}$ & 0.4360 & 0.5819 & 0.3214 & 0.06019 \\
\hline \multirow{4}{*}{$\begin{array}{c}\text { TD-TMD } \\
\text { (geometrically } \\
\text { nonlinear) }\end{array}$} & $R_{X}$ & 0.5023 & 0.9610 & 0.3018 & 0.15256 \\
\hline & $R_{Y}$ & 0.5469 & 0.9039 & 0.3002 & 0.14870 \\
\hline & $R_{Z}$ & 0.3277 & 0.4258 & 0.2533 & 0.04319 \\
\hline & $R_{X Y Z}$ & 0.4360 & 0.5832 & 0.3218 & 0.06021 \\
\hline \multirow{4}{*}{$\begin{array}{l}\text { SD-TMD } \\
\text { (Case 1) }\end{array}$} & $R_{X}$ & 0.4423 & 0.5647 & 0.3647 & 0.06600 \\
\hline & $R_{Y}$ & 0.4423 & 0.5647 & 0.3647 & 0.06600 \\
\hline & $R_{Z}$ & 0.4203 & 0.4613 & 0.3546 & 0.03952 \\
\hline & $R_{X Y Z}$ & 0.4289 & 0.4742 & 0.3787 & 0.02478 \\
\hline \multirow{4}{*}{$\begin{array}{l}\text { SD-TMD } \\
\text { (Case 2) }\end{array}$} & $R_{X}$ & 0.4501 & 0.5920 & 0.3720 & 0.07424 \\
\hline & $R_{Y}$ & 0.4501 & 0,5920 & 0.3720 & 0.08424 \\
\hline & $R_{Z}$ & 0.4285 & 0.4702 & 0.3696 & 0.03928 \\
\hline & $R_{X Y Z}$ & 0.4367 & 0.4864 & 0.3875 & 0.02643 \\
\hline \multirow{4}{*}{$\begin{array}{l}\text { SD-TMD } \\
\text { (Case 3) }\end{array}$} & $R_{X}$ & 0.4368 & 0.5620 & 0.3637 & 0.06632 \\
\hline & $R_{Y}$ & 0.4368 & 0.5620 & 0.3637 & 0.06632 \\
\hline & $R_{Z}$ & 0.4224 & 0.4705 & 0.3538 & 0.04255 \\
\hline & $R_{X Y Z}$ & 0.4263 & 0.4701 & 0.3755 & 0.02499 \\
\hline \multirow{4}{*}{$\begin{array}{l}\text { SD-TMD } \\
\text { (Opt.) }\end{array}$} & $R_{X}$ & 0.4325 & 0.5380 & 0.3586 & 0.05825 \\
\hline & $R_{Y}$ & 0.4325 & 0.5380 & 0.3586 & 0.05825 \\
\hline & $R_{Z}$ & 0.4102 & 0.4656 & 0.3219 & 0.05031 \\
\hline & $R_{X Y Z}$ & 0.4192 & 0.4714 & 0.3627 & 0.02563 \\
\hline
\end{tabular}


To check the robustness of the TD-TMD and SD-TMD, response reduction ratios against 60 sets of motions, which are compatible to the response spectrum in Fig. 4, are listed in Table 7. Note that the motions in Z-direction are scaled by 0.5 also in this case. It is seen from the table that the TD-TMD has almost the same performance in $R_{X Y Z}$ as all cases including Cases 1-3 and optimal solution indicated by 'Opt.' of SD-TMDs with the same total mass. However, the TD-TMD has larger standard deviation than the SD-TMDs, and the maximum value among 60 waves of the TD-TMD may have large values in $X$ - and $Y$ directions.

Geometrically nonlinear analysis is carried out for the optimal TD-TMD to check the effect of deformation during vibration. It is seen from Table 7 that the effect of geometrical nonlinearity is very small. Note that the maximum relative displacements of node D from node A in $X$-, $Y$-, and $Z$-directions are $0.0189,0.0139$, and $0.0170 \mathrm{~m}$ under the seismic motion in Fig. 3. These values are sufficiently smaller than the size of TDTMD; e.g., the length of viscous damper is $1 \mathrm{~m}$.

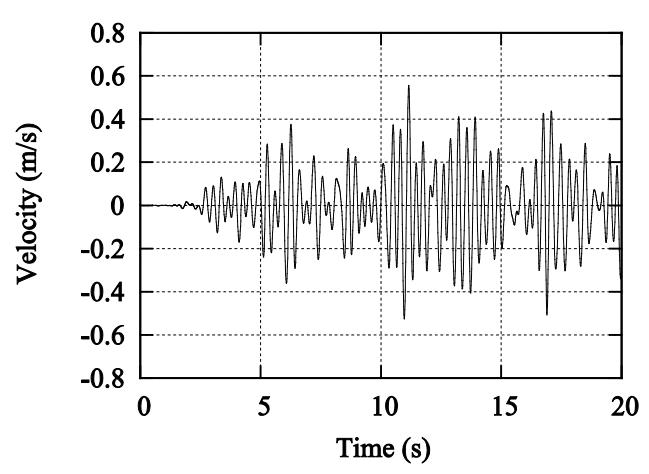

(a)

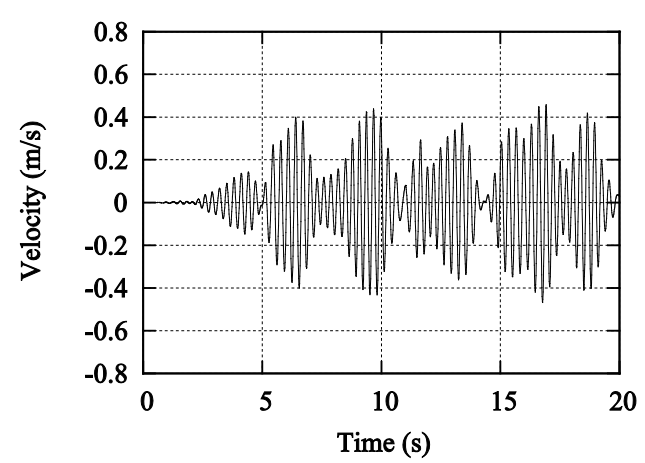

(c)

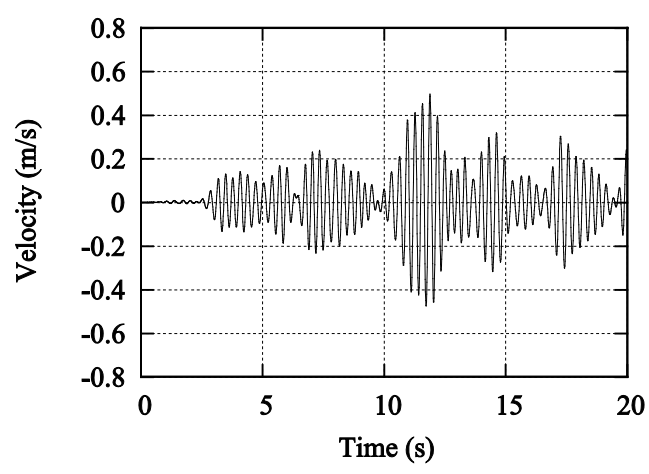

(b)

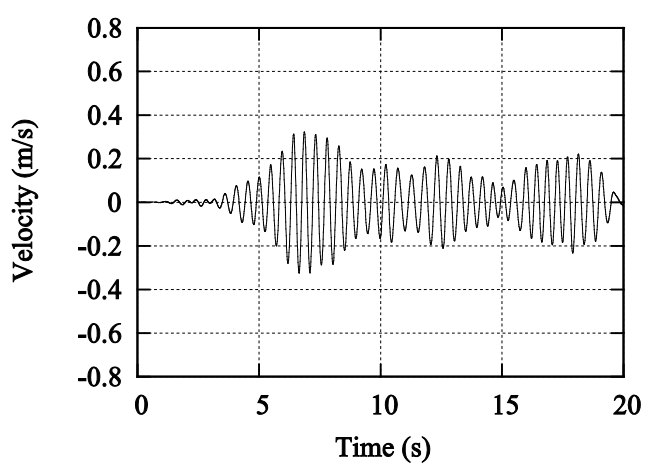

(d) 
Figure 7: Time-histories of velocity of damper; (a) TD-TMD, (b) SD-TMD ( $X$ direction), (c) SD-TMD ( $Y$-direction), (d) SD-TMD (Z-direction).

Time-histories of velocity of dampers are plotted in Fig. 7 for the first 20 seconds of seismic excitation. Velocity of the damper of TD-TMD is similar to those in $X$ - and $Y$ directions of SD-TMD, and the damper of TD-TMD dissipates energy constantly during earthquake excitation as demonstrated later.

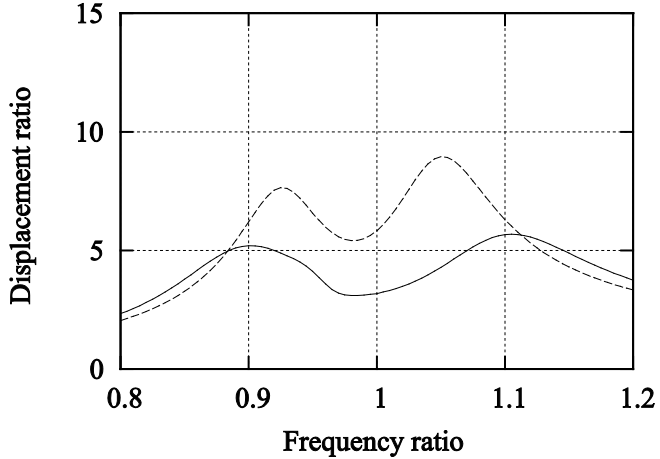

(a)

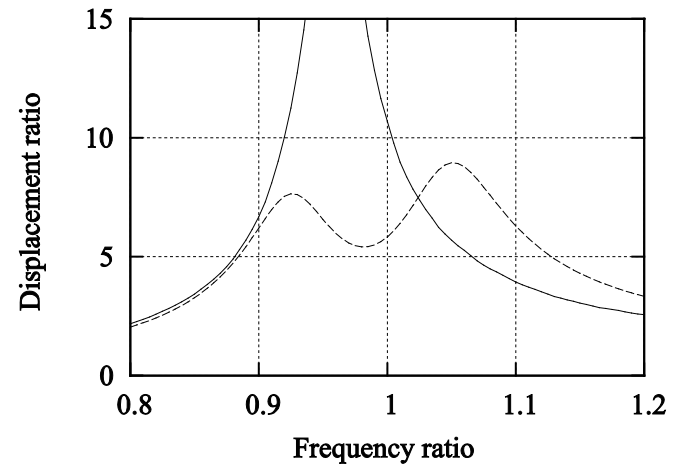

(b)

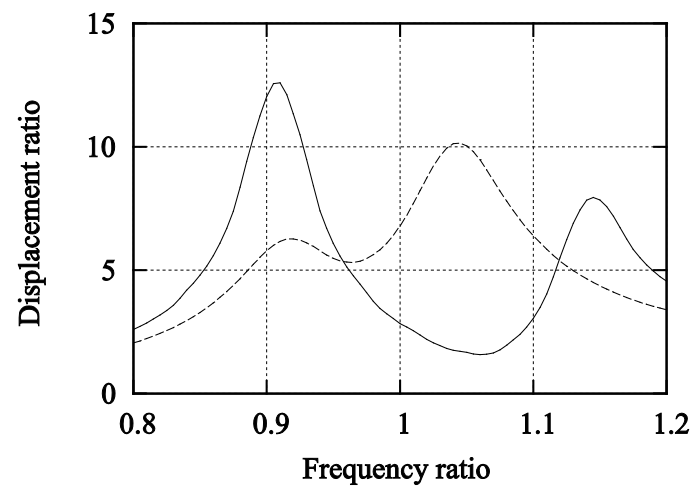

(c)

Figure 8: Displacement amplification ratios of the structure; solid line: TD-TMD, dashed line SD-TMD; (a) $X$-direction, (b) $Y$-direction, (c) $Z$-direction.

Displacement amplification ratio is defined as the ratio of maximum absolute value of displacement of the structure (node A in Fig. 5) to the magnitude of displacement of sinusoidal excitation. The displacement amplification ratios for the inputs in $X-, Y$-, and $Z$-directions are plotted in Fig. 8 for structures with TD-TMD and SD-TMD, where the frequency ratio is the ratio of frequency of the excitation to the natural frequency of the structure in each direction. If the frequency ratio is close to 1 , the TD-TMD has better performance than SD-TMD in $X$ - and $Z$-directions, but has larger displacement ratio in $Y$ - 
direction. Note that the vibration of TD-TMD in three-direction interacts with each other; therefore, the performance of TD-TMD cannot be discussed from the reduction ratio independently in each direction.

The time-histories of displacement of the structure and the relative displacement of mass of the optimal TD-TMD from the structure are plotted in Figs. 9(a)-(c) in solid and dashed lines, respectively. The responses from 10 to 20 seconds are plotted to closely inspect the phase difference. The time-histories of displacements for the optimal SDTMD in $X$-direction are also plotted in Fig. 9(d). As sheen from Fig. 9, the relative displacement of the TD-TMD is mainly delayed from the displacement of the structure to exhibit the typical property of a TMD.

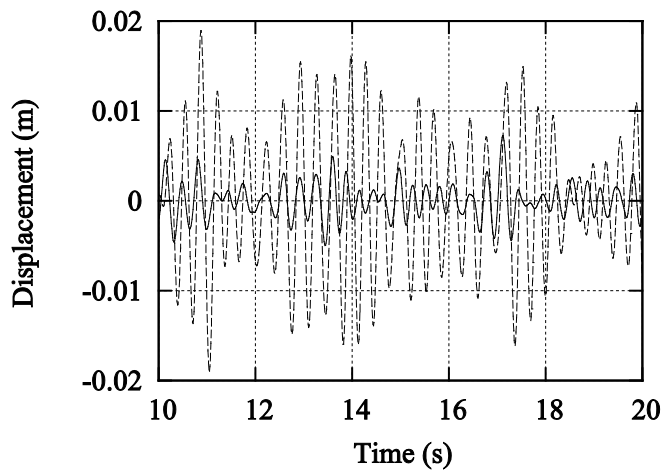

(a)

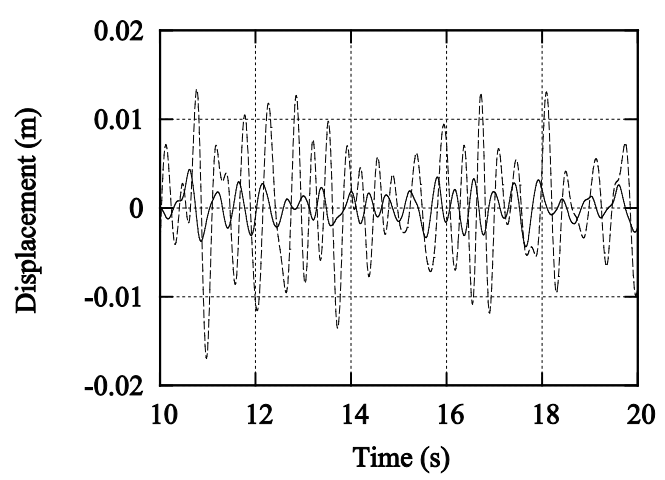

(c)

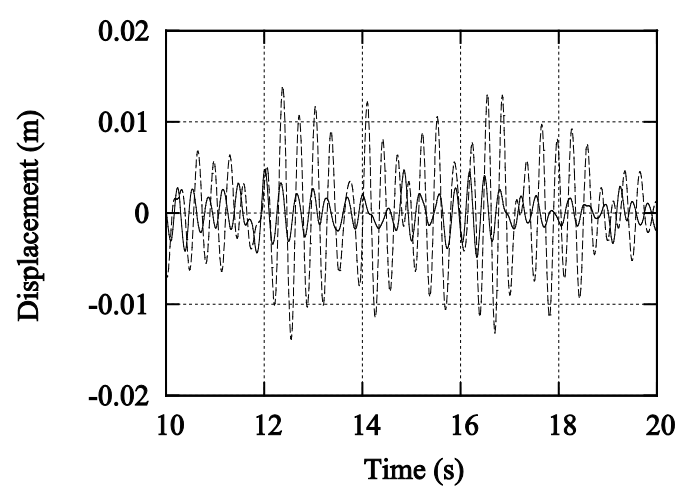

(b)

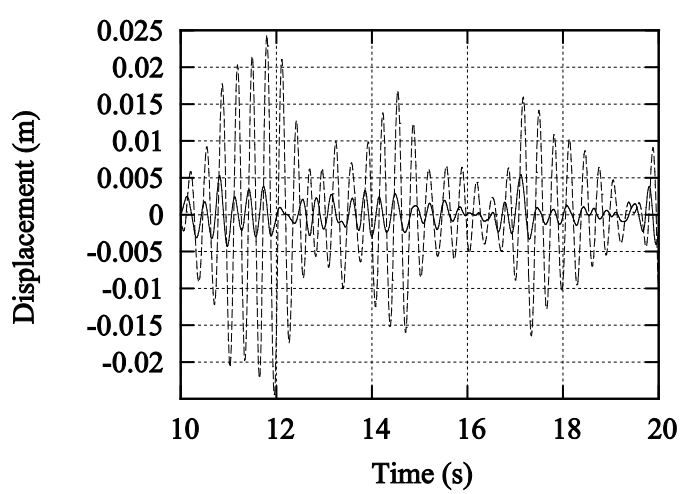

(d)

Figure 9: Time-histories of displacements of structure (solid line) and relative displacement of mass of TMD from structure (dashed line); (a) TD-TMD, $X$-direction,

(b) TD-TMD, $Y$-direction, (c) TD-TMD, $Z$-direction, (d) SD-TMD, $X$-direction. 


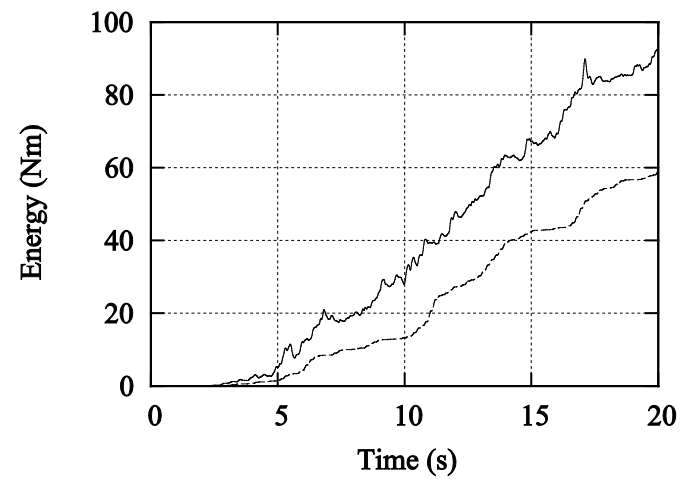

Figure 10: Time histories of input energy (solid line) and dissipated energy by TDTMD (dashed line).

The accumulated input energy and dissipated energy by the damper of TD-TMD are plotted in solid and dashed lines, respectively, in Fig. 10. As seen from the figure, the dissipated energy increases in a similar manner as the input energy, and more than $60 \%$ of the energy is dissipated by the damper.

Table 9: Response reduction ratios for recorded motions.

\begin{tabular}{cccccc}
\hline & & El Centro & Hachinohe & Taft & Tohoku \\
\hline \multirow{2}{*}{ Response } & $D_{X}$ & 1.151 & 0.418 & 0.744 & 0.418 \\
$\left(\times 10^{-5}\right)$ & $D_{Y}$ & 0.164 & 0.591 & 0.281 & 1.231 \\
& $D_{Z}$ & 19.352 & 5.560 & 1.893 & 11.654 \\
\hline & $R_{X}$ & 0.561 & 1.128 & 0.352 & 1.326 \\
TD-TMD & $R_{Y}$ & 1.585 & 0.791 & 0.774 & 0.624 \\
& $R_{Z}$ & 0.214 & 0.329 & 0.456 & 0.383 \\
& $R_{X Y Z}$ & 0.244 & 0.421 & 0.460 & 0.435 \\
SD-TMD & $R_{X}$ & 0.519 & 0.831 & 0.506 & 0.831 \\
$($ Opt.) & $R_{Y}$ & 0.480 & 0.538 & 0.449 & 0.547 \\
& $R_{Z}$ & 0.256 & 0.401 & 0.614 & 0.531 \\
& $R_{X Y Z}$ & 0.272 & 0.440 & 0.571 & 0.541 \\
\hline
\end{tabular}

To confirm the performance of TD-TMD for recorded motions, response reduction ratios are computed for the waves El Centro 1940, Hachinohe 1968, Taft 1952, and Tohoku 1978. Note that Hachinohe wave is used as a typical far field ground motion in seismic design process in Japan. Since the optimal TD-TMD has better response reduction performance in $X$-and $Z$-directions than in $Y$-direction, the EW, NS, and UD components 
are applied in $X$-, $Z$-, and $Y$-directions, respectively, of the TD-TMD; i.e., the damper of the TD-TMD is assumed to be located in the horizontal plane.

The response reduction ratios as well as the mean square norm of the displacement are listed in Table 9, which shows that TD-TMD has better performance in $R_{X Y Z}$ for all four waves. Note that the largest value among $R_{X}, R_{Y}$, and $R_{Z}$ of TD-TMD corresponds to the direction that has the smallest value among $D_{X}, D_{Y}$, and $D_{Z}$, which means that the direction with small displacement is sacrificed to obtain small value of $R_{X Y Z}$ of the displacement norm.

\section{Conclusions}

A mass damper called TD-TMD has been proposed for reduction of seismic responses in three directions using a set of single mass and viscous damper. The conclusion obtained from this study is summarized as follows:

1. The three-directional response of a structure can be reduced using a tuned mass damper called TD-TMD that has a tetrahedral shape. The TD-TMD consists of a mass, viscous damper, three springs, and a rigid bar.

2. The parameters of TD-TMD can be tuned by solving an optimization problem for minimizing the mean value of response reduction ratios against specified five sets of seismic motions, which are compatible to the specified design response acceleration spectrum. The bounds of variables are defined by solving an auxiliary static optimization problem to maximize the minimum damper deformation under unit static loads in various directions, where static stiffness in three-directions are constrained to be equal to the optimal stiffness of a conventional SD-TMD assigned in each direction.

3. Since the dynamic optimization problem to minimize the mean response reduction ratio is highly nonlinear, the pure random search followed by the heuristic algorithm called simulated annealing is very effective to obtain efficient local optimal solutions.

4. The performance of the proposed TD-TMD has been confirmed in comparison to the three conventional SD-TMDs assigned in each direction of a simple 3DOF structure representing three dominated modes of a general structure. TD-TMD has an equivalent performance with SD-TMDs, although the frequency responses of TD- 
TMD and SD-TMD in three-directions are different. It has also been confirmed that TD-TMD has better performance than SD-TMD for recorded ground motions.

5. The performance of TD-TMD has also been confirmed from the energy dissipation property and the phase difference between the structural displacement and the relative displacement between the structure and the mass, which exhibits typical characteristics of a TMD.

\section{References}

1. Lin CC, Wang JF, Lien CH, Chiang HW, Lin CS. Optimum design and experimental study of multiple tuned mass dampers with limited stroke. Earthquake Eng Struct Dyn 2010; 39: 1631-1651.

2. Chen $\mathrm{G}, \mathrm{Wu}$ J. Optimal placement of multiple tuned mass dampers for seismic structures. T. Struct Eng 2001; 127: 1054-1062.

3. Viet LD, Nghi NB. On a nonlinear single-mass two-frequency pendulum tuned mass damper to reduce horizontal vibration. Eng Struct 2014; 81: 175-180.

4. Ueng JM, Lin CC, Wang JF. Practical design issues of tuned mass dampers for torsionally coupled buildings under earthquake loadings. Struct Design Tall Spec Build 2008; 17, 133-165.

5. Yoshinaka S, Kawaguchi K. A study on spatially dispersed arranged TMDs based on MTMD method for vibration control of plural modes of large span structures. J Struct Constr Eng, AIJ 2004; 586, 123-130. (in Japanese)

6. Tsuda S, Ohsaki M. Parameter optimization of mass damper consisting of compliant mechanism for bi-directional control of spatial structures. J Struct Constr Eng, AIJ 2012(673): 379-387. (in Japanese)

7. Tsuda S, Ohsaki M. Bi-directional seismic vibration control of spatial structures using passive mass damper consisting of compliant mechanism. Proc. IASS-APCS 2012. Seoul. Paper No. 109.

8. Almazán JL, De la Llera JC, Inaudi JA, López-Garc í a D, Izquiardo LE. A bidirectional and homogeneous tuned mass damper: A new passive control of vobrations. Eng Struct 2007; 29: 1548-1560.

9. De Angelis M, Perno S, Reggio A. Dynamic response and optimal design of structures with large mass ratio TMD. Earthquake Eng Struct Dyn 2012; 41: 41-60. 
10. Lavan O, Daniel Y. Full resources utilization seismic design of irregular structures using multiple tuned mass dampers. Structural and Multidisciplinary Optimization 2013; 48(3): 517-532

11. Lin JL, Tsai KC, Yu YJ. Bi-directional coupled tuned mass dampers for the seismic response control of two-way asymmetric-plan buildings. Earthquake Eng Struct Dyn 2011; 40: 675-690.

12. Garrido H, Curadeli O, Ambrosini D. Improvement of tuned mass damper by using rotational inertia through tuned viscous mass damper. Eng Struct 2013; 56: 2149-2153.

13. Bekdaş G, Nigdeli S. Estimating optimum parameters of tuned mass dampers using harmony search. Eng Struct 2011; 33: 2716-2723.

14. Leung AYT, Zhang H. Particle swarm optimization of tuned mass dampers. Eng Struct 2009; 31: 715-728.

15. Zhigljavsku A, Zilinskas A. Stochastic Global Optimization 2010, Springer.

16. Ohsaki M, Katsura M. A random sampling approach to worst-case design of structures. Struct Multidisc Optim 2012; 46, 27-39.

17. Kirkpatrick S, Gelatt CD, Vecchi MP. Optimization by simulated annealing. Science 1983; 220(4595), 671-680.

18. Goffe WL, Ferrier GD, Gogers J. Global optimization of statistical functions with simulated annealing. J Econometrics 1994; 60, 65-99.

19. Hartog JPD. Mechanical Vibrations 1985; Dover Publications.

20. Open System for Earthquake Engineering Simulation (OpenSees), PEERC, UC Berkeley. http://opensees.berkeley.edu/, August 1, 2015.

21. Scanlan RH, Sachs K. Earthquake time histories and response spectra. J Eng Mech Div, ASCE 1974; EM4: 635-655.

22. Iyenger RN, Rao PN. Generation of spectrum compatible accelerograms. Earthquake Eng Struct Dyn 1979; 7: 253-263.

23. Gill PE, Murray W, Saunders MA. SNOPT: An SQP algorithm for large-scale constrained optimization. SIAM J Optim 2002;12, 979-1006. 